\title{
Risk Factors for Bloodstream Infections Among an Urban Population with Skin and Soft Tissue Infections: A Retrospective Unmatched Case-Control Study
}

\author{
Michael J. Rybak · Evan J. Zasowski - Sarah C. J. Jorgensen • \\ Abdalhamid M. Lagnf · Susan L. Davis · Aaron B. Mendelsohn • \\ Vasileios Margaritis
}

Received: October 29, 2018 / Published online: December 18, 2018

(C) The Author(s) 2018

\section{ABSTRACT}

Introduction: The prevalence of acute bacterial skin and skin structure infections (ABSSSIs) continues to increase. Bloodstream infection (BSI) is a severe secondary complication of ABSSSI. The objective of this study was to determine clinical and sociodemographic risk factors for BSI in patients with acute bacterial skin and skin structure infections (ABSSSIs) and

Enhanced digital features To view enhanced digital features for this article go to https://doi.org/10.6084/ m9.figshare.7416182.

M. J. Rybak (ه) · E. J. Zasowski · S. C. J. Jorgensen · A. M. Lagnf · S. L. Davis

Anti-Infective Research Laboratory, College of Pharmacy and Health Sciences, Wayne State

University, Detroit, MI, USA

e-mail:m.rybak@wayne.edu

\section{J. Rybak}

Department of Pharmacy, Detroit Medical Center, Detroit, MI, USA

M. J. Rybak

School of Medicine, Wayne State University,

Detroit, MI, USA

A. B. Mendelsohn · V. Margaritis

College of Health Sciences, Walden University,

Minneapolis, MN, USA

S. L. Davis

Department of Pharmacy, Henry Ford Hospital, Detroit, MI, USA to determine if sociodemographic factors impact severity at presentation.

Methods: This was a retrospective unmatched (1:1) case-control study. Predictors of BSI and severe infection were sought through multivariable logistic regression analyses. Cases and controls were collected from two major medical centers located in downtown Detroit, Michigan: the Detroit Medical Center and the Henry Ford Health System. The population of interest included adult patients with community-onset (CO) ABSSSI treated at a participating hospital between January 2010 and December 2015. Cases were defined as those developing BSI within $48 \mathrm{~h}$ of admission with CO-ABSSSI as the primary source, while controls were those with CO-ABSSSI without BSI.

Results: A total of 392 patients (196 cases, 196 controls) were included. Independent predictors of BSI were male gender (aOR 1.85: 95\% CI $1.11,3.66$ ), acute renal failure (aOR 2.08: 95\% CI 1.18, 3.66), intravenous drug use (aOR 4.38, $95 \%$ CI 2.22, 8.62), and prior hospitalization (aOR 2.41, 95\% CI 1.24, 4.93). African American race (aOR 2.18, 95\% CI 1.38, 3.4), leukocytosis (aOR 2.24, 95\% CI 1.41, 3.55), and prior hospitalization (aOR 2.07, 95\% CI 1.19, 3.00) were significantly associated with infection severity. Conclusion: Both clinical and sociodemographic factors were associated with BSI and severe infection underscoring the importance of social determinants of health in outcomes among underserved populations. 
Keywords: Acute bacterial skin and skin structure infections; Bloodstream infection; Public health

\section{INTRODUCTION}

Acute bacterial skin and skin structure infections (ABSSSIs) are complicated skin and skin structure infections that generally require hospitalization and parenteral antibiotics and include the diagnosis of cellulitis/erysipelas, wound infection, major cutaneous abscess with an accompanying redness, or edema that extends to a minimum lesion surface area of $75 \mathrm{~cm}[2,1]$. ABSSSIs are among the most common infections encountered in community and healthcare settings [2,3]. The number of patients diagnosed with ABSSSIs has steadily increased in the USA over the last 2 decades [4-6]. This increase in ABSSSIs has coincided with rising hospital admissions and complications including secondary bone and joint infections, amputations, and bloodstream infections (BSI) $[7,8]$. BSI, although still relatively rare $(5-12 \%)[9,10]$, is considered one of the most severe complications of ABSSSI as it carries a significant risk for morbidity and mortality $[9,11]$. The most common bacterial pathogen associated with BSI secondary to ABSSSI is Staphylococcus aureus. Although once restricted to patients with traditional healthcare-associated risk factors, community-associated methicillin-resistant $S$. aureus (CA-MRSA) is now a predominant pathogen in ABSSSI, and prevalence is typically higher in urban populations, disproportionately affecting individuals of low socioeconomic status $[12,13]$.

Because secondary BSI in ABSSSI is associated with high rates of mortality, several investigators have attempted to identify patient characteristics that are associated with this complication [9, 14, 15]. However, risk factors for BSI have not been evaluated in African American patients with ABSSSI and low socioeconomic status in an urban setting. Therefore, the primary aim of this study was to identify significant clinical and sociodemographic risk factors for BSI in patients with community-onset (CO) ABSSSI residing in downtown Detroit.
In addition, we sought to determine the extent to which clinical and sociodemographic factors impact severity of infection in patients with ABSSSI + BSI.

\section{METHODS}

\section{Study Setting and Patient Population}

This retrospective, case-control study was conducted at two major medical centers located in downtown Detroit, Michigan: the Detroit Medical Center (DMC) and the Henry Ford Health System (HFHS). Both medical centers have centrally located campuses and therefore service a large section of the inner-city Detroit patient population. The population of interest consisted of patients hospitalized with COABSSSI diagnosed within $48 \mathrm{~h}$ of admission, as previously defined [16] between January 2010 and December 2015. Patients were excluded if they had any of the following concomitant diagnoses because these infections tend to have a higher likelihood of poor patient outcomes irrespective of concomitant BSI: [17] osteomyelitis, septic arthritis, prosthetic devicerelated infection, an animal or human bite, burn, necrotizing fasciitis, pyomyositis, or ABSSSI secondary to recent (within 2 weeks) surgery. Cases were defined as inpatients with at least one positive blood culture meeting the Center for Disease Control and Prevention (CDC) BSI criteria [18] collected within $48 \mathrm{~h}$ of admission and in whom ABSSSI was documented as the primary source. Study controls consisted of inpatients with CO-ABSSSI and blood cultures collected within $48 \mathrm{~h}$ of admission demonstrating no microbiologic evidence of BSI.

\section{Data Collection and Definitions of Study Variables}

Both case and control patients were identified using International Classification of Diseases, Ninth Revision, Clinical Modification [ICD-9$\mathrm{CM}$ ] diagnostic codes via the DMC and HFHS electronic medical records (EMRs). Data 
elements including demographics, comorbid conditions, prior hospitalization (180 days), history of $S$. aureus infection (60 days), prior ABSSSI (60 days), recent antibiotic exposure (30 days), vital signs (using the worst physiologic values within $24 \mathrm{~h}$ of admission), laboratory values, microbiology and antibiotic treatment were extracted from the EMR. All cases were screened and entered in the secure database, REDCap (Research Electronic Data Capture) [19], an electronic data capture tool hosted by Wayne State University by a trained research assistant. Relevant data in the EMR were used to determine whether the patient met the criteria for ABSSSI according to FDA definitions.

Income and education levels were derived from the Census Bureau of Statistics by the patient's zip code. Severe ABSSSI was defined as need for incision and drainage or wound debridement in the operating room, amputation, admission to the intensive care unit (ICU) or severe sepsis, as previously defined [20]. Complications secondary to ABSSSI infection were defined as the development of endocarditis, osteomyelitis, and/or other sites of metastatic infection not present on admission. Thirty-day post-discharge vital status was ascertained from in- and outpatient records and categorized as deceased or alive with or without readmission. Patients with no follow-up visits or readmissions within 30 days of discharge were assumed to be alive without readmission. Readmission was considered ABSSSI-related based on physician documentation. This study was approved by the institutional review boards (IRBs) at Walden University (IRB\# 0303767) and Wayne State University (IRB\# 010616M1E), with the latter serving as coordinating IRB for the DMC review committee and HFHS IRB. A waiver of informed consent was granted. The study was performed in accordance with the 1964 Helskinki Declaration and its later amendments.

\section{Statistical Analysis}

The a priori minimum sample size for this unmatched 1:1 case-control study was calculated using the statistical test for differences in proportions [21]. A sample size of 187 case and 187 control subjects was required to achieve $80 \%$ power at a 0.05 significance level to detect an adjusted odds ratio (aOR) of $\geq 2$ for a risk factor with a known prevalence of $10-20 \%$ (diabetes, renal failure, prior antibiotics, recurrent infection) [17, 22-24].

Descriptive statistics were used to characterize cases and controls. Continuous variables were compared by Student's $t$ test if normally distributed and Mann-Whitney $U$ test if the normality assumption was not met. Categorical variables were evaluated using Pearson's chisquare test or a two-tailed Fisher's exact test, as appropriate. Explanatory variables that were associated with case status $(P \leq 0.1)$ in the unadjusted analyses were included in a multivariable logistic regression model. A backwardsstepwise elimination approach was used to eliminate the variable with the largest $P$ value at each step. The Hosmer-Lemeshow statistic was used to evaluate the predicted and observed probability to test for evidence of a lack of a model fit [25]. Relationships between the explanatory variables and BSI were evaluated using the odds ratio (OR) and corresponding 95\% confidence interval (CI). An identical procedure was used to identify a set of risk factors independently associated with increased risk of severe ABSSSI. All calculations were performed using IBM SPSS Statistics software, version 22.0 (IBM SPSS, Armonk, NY).

\section{RESULTS}

A total of 5267 patient records were screened for inclusion during the study period and 392 met criteria for inclusion (196 cases and 196 controls). Among potential cases $(n=3868)$, reasons for exclusion included the primary BSI source other than skin/soft tissue $(n=1579)$, excluded skin infection (chronic ulcer, burn, deep abscess, gangrene, necrotizing fasciitis; $n=1102)$, hemodialysis $(n=367)$, prosthetic material $\quad(n=210), \quad$ immunosuppression $(n=120)$, BSI $>48 \mathrm{~h}$ after admission $(n=249)$, and pregnancy $(n=45)$. Among potential controls $(n=1399)$, reasons for exclusion included 
concomitant infection $(n=288)$, excluded skin infection $(n=610)$, hemodialysis $(n=158)$, prosthetic material $(n=82)$, immunosuppression $(n=55)$, and pregnancy $(n=10)$. Demographics, sociodemographics, clinical characteristics and outcomes of cases and controls are shown in Table 1. The mean age of the overall population was 48.7 (SD 17.4) years with a male predominance $(59.2 \%)$. With respect to race, African Americans made up the majority of the study population at $56.1 \%$ followed by Caucasians at $37.2 \%$. In terms of sociodemographic characteristics by zip code, $6.9 \%$ had resided in a zip code with $>30 \%$ less than a high school education, $35.7 \%$ were considered below the federal poverty level in terms of median household income, and $19.1 \%$ had no medical insurance.

On univariate analysis, cases (ABSSSI + BSI) were significantly older $(P<0.001)$ and predominately male $(P=0.008)$ compared with controls. No significant differences were observed regarding race or sociodemographic characteristics by zip code such as education, median income levels, or the type or presence of medical insurance (Table 1). As indicated by the median Charlson Comorbidity Index, case patients tended to have more underlying comorbid conditions such as chronic renal failure and diabetes compared with controls $(P<0.001)$. The most common underlying conditions were diabetes, hypertension, and intravenous drug use. In addition, there were significantly more cases with previous hospitalization $(P<0.001)$ and prior infection with methicillin-susceptible $S$. aureus (MSSA) $(P=0.014)$. Case patients also had a higher percentage of abnormal temperature, elevated white blood cell count (WBC), and acute renal failure on admission $(P<0.001)$. In addition, cellulitis and infected wounds or ulcers were more common among cases whereas controls had a higher percentage of abscess or abscess plus cellulitis $(P<0.001)$. Although there was no difference in the infection site (e.g., upper versus lower extremity, $P=0.930)$, controls had a higher rate of MRSA $(P=0.004)$ (Table 1$)$.

Vancomycin was the most commonly used antibiotic overall. As shown in Fig. 1, a significantly higher percentage of patients with
ABSSSI + BSI were treated with vancomycin compared with those with ABSSSI (79.1\% vs. $64.3 \%, P<0.001)$. Clindamycin was the second most common antibiotic administered. Although the use of clindamycin was higher in patients with ABSSSI, this difference was not statistically significant $(27 \%$ vs. $18.9 \%$, $P=0.055)$. Cephalexin use was significantly higher in patients with ABSSSI; however, the overall use of this agent was low ( $6 \%$ vs. $0 \%$, $P=0.030$ ).

A higher percentage of cases had an infectious disease consult and were admitted to the ICU $(P<0.001)$. Length of stay was also significantly longer for cases (7.4 days vs. 2.7 days, $P<0.001)$. Secondary complications, clinical failure at discharge, and re-infection within 30 days of discharge were more common in case patients $(P=0.034, \quad P=0.003, \quad P=0.006$, respectively). Although in-hospital mortality was low overall $(2 \%)$, all deaths occurred among case patients $(P<0.001)$.

Table 2 displays the results of the multivariable logistic regression analysis. Variables associated with ABSSSI + BSI were male gender (aOR 1.85, 95\% CI 1.11, 3.66), acute renal failure (aOR 2.08, 95\% CI 1.18, 3.67), intravenous drug use (aOR 4.38, 95\% CI 2.22, 8.62), prior hospitalization (aOR of $2.41,95 \%$ CI $1.24,4.93$ ), abnormal temperature (aOR 2.86, 95\% CI 1.66, 4.93), and elevated WBC (aOR 4.26, 95\% CI $2.43,7.47)$. Abscess or abscess and cellulitis were protective against ABSSSI + BSI (aOR of 0.220, 95\% CI 0.11, 0.46 and aOR 0.139, 95\% CI 0.07, 0.28 , respectively). The Hosmer-Lemeshow goodness of fit test was not significant $(P=0.305)$, indicating that there was no evidence of a lack of model fit.

A total of 166 patients (42.3\%) met the criteria for severe ABSSSI. Regarding risk factors for severe ABSSSI, African American race (OR 2.17, $95 \%$ CI 1.43, 3.28), elevated WBC count (OR 2.32, 95\% CI 1.52, 4.50), abscess and cellulitis (OR 3.0, 95\% CI 1.39, 3.17), recent antibiotic exposure (OR 2.07 95\% CI 1.02, 4.24), and prior MRSA infection (OR 2.857 95\% CI 1.05, 7.77) were associated with severe ABSSSI on univariate analysis.

Table 3 displays the results of the multivariable logistic regression analysis for severe 
Table 1 Demographics, sociodemographic, clinical characteristics, and outcomes of cases and controls, Detroit, MI, 2010-2015

\begin{tabular}{|c|c|c|c|}
\hline Variables & ABSSSI + BSI $(n=196)$ & ABSSSI $(n=196)$ & $P$ value \\
\hline Age (years), mean (SD) & $52.4(18.4)$ & $45.3(15.5)$ & $<0.001$ \\
\hline Gender, $n,(\%)$ & & & 0.008 \\
\hline Male & $129(65.8)$ & $103(52.6)$ & \\
\hline Female & $67(34)$ & $93(47.0)$ & \\
\hline Race, $n,(\%)$ & & & 0.520 \\
\hline African-American & $112(57.1)$ & $108(55.1)$ & \\
\hline Asian & $1(0.5)$ & $1(0.5)$ & \\
\hline Caucasian & $68(34.7)$ & $78(39.8)$ & \\
\hline Other & $15(7.7)$ & $9(4.6)$ & \\
\hline $\mathrm{BMI}>30 \mathrm{~kg} / \mathrm{m}^{2}, n,(\%)$ & $79(41.6)$ & $91(46.4)$ & 0.340 \\
\hline \multicolumn{4}{|l|}{ Sociodemographics, $n$, (\%) } \\
\hline Education $>30 \%<$ high school $^{\mathrm{a}}$ & $18(9.2)$ & $9(9.6)$ & 0.073 \\
\hline Median income $<$ poverty $^{\mathrm{b}}$ & $78(39.8)$ & $62(31.6)$ & 0.092 \\
\hline No medical insurance, $n,(\%)$ & $39(19.9)$ & $36(18.4)$ & 0.399 \\
\hline \multicolumn{4}{|l|}{ Comorbid conditions } \\
\hline Chronic renal failure, $n$, (\%) & $25(12.9)$ & $8(4.1)$ & 0.002 \\
\hline Hepatic disease, $n$, (\%) & $18(9.2)$ & $4(2.6)$ & 0.005 \\
\hline Diabetes, $n$, (\%) & $76(38.8)$ & $49(25.0)$ & 0.003 \\
\hline Paraplegia, $n,(\%)$ & $4(2)$ & $0(0)$ & 0.440 \\
\hline Hypertension, $n$, (\%) & $88(44.9)$ & $62(31.6)$ & 0.007 \\
\hline Congestive heart failure, $n,(\%)$ & $19(9.7)$ & $7(3.6)$ & 0.015 \\
\hline IV drug abuse, $n$, (\%) & $44(22.4)$ & $25(12.8)$ & 0.012 \\
\hline Charlson Comorbidity Index, median, (IQR) & $2(1-5)$ & $1(0-3)$ & $<0.001$ \\
\hline Prior hospitalization 180 days, $n,(\%)$ & $63(32.1)$ & $25(12.8)$ & $<0.001$ \\
\hline Prior antibiotic use past 30 days, $n(\%)$ & $18(9.2)$ & $16(8.2)$ & 0.720 \\
\hline Prior MSSA infection 60 days, $n$, (\%) & $6(3.1)$ & $0(0)$ & 0.014 \\
\hline Prior MRSA infection 60 days, $n,(\%)$ & $11(5.6)$ & $7(3.6)$ & 0.334 \\
\hline History of ABSSSI past 60 days, $n,(\%)$ & $22(11.2)$ & $14(7.1)$ & 0.162 \\
\hline Days from symptom onset to admission, median $(\mathrm{IQR})^{\mathrm{c}}$ & $5(3,7)$ & $5(2,9)$ & 0.358 \\
\hline Abnormal temperature ${ }^{\mathrm{d}}, n,(\%)$ & $87(44.4)$ & $38(19.4)$ & $<0.001$ \\
\hline Elevated $\mathrm{WBC}^{\mathrm{e}}, n,(\%)$ & $138(60.3)$ & $91(39.7)$ & $<0.001$ \\
\hline Acute renal failure, $n(\%)$ & $98(50)$ & $48(24.5)$ & $<0.001$ \\
\hline Type of ABSSSI, $n(\%)$ & & & $<0.001$ \\
\hline Abscess & $56(28.6)$ & $74(37.8)$ & \\
\hline Cellulitis & $56(28.6)$ & $20(10.2)$ & \\
\hline Abscess + cellulitis & $58(29.6)$ & $98(50)$ & \\
\hline Infected wound & $24(12.2)$ & $4(2)$ & \\
\hline Ulcer & $2(1)$ & $0(0)$ & \\
\hline Infection site, $n,(\%)$ & & & 0.930 \\
\hline Upper extremity & $46(23.5)$ & $65(33.2)$ & \\
\hline Lower extremity & $84(42.9)$ & $83(42.3)$ & \\
\hline Head/neck & $31(15.9)$ & $20(10.2)$ & \\
\hline Torso/trunk & $35(17.9)$ & $28(14.3)$ & \\
\hline Methicillin-resistant $S$. aureus, $n$, (\%) & $126(64.3)$ & $152(77.7)$ & 0.004 \\
\hline Polymicrobial $^{\mathrm{f}}, n,(\%)$ & $21(10.8)$ & $22(11.2)$ & 0.886 \\
\hline
\end{tabular}


Table 1 continued

\begin{tabular}{lcrr}
\hline Variables & ABSSSI + BSI $(\boldsymbol{n}=\mathbf{1 9 6})$ & ABSSSI $(\boldsymbol{n}=\mathbf{1 9 6})$ & $\boldsymbol{P}$ value \\
\hline Clinical outcomes & & & $<4(27.6)$ \\
ID consult & $115(58.7)$ & $5(2.6)$ & $<0.001$ \\
Admission to ICU, $n$, (\%) & $25(12.8)$ & $2.7(2.2)$ & $<0.001$ \\
Length of hospital stay (days), mean, (SD) & $7.4(5.7)$ & $7(3.6)$ & $<0.001$ \\
Clinical failure at discharge, , $n,(\%)$ & $22(11.3)$ & $0(0)$ & 0.003 \\
In-hospital mortality, $n$ (\%) & $8(4.1)$ & $1(0.5)$ & $<0.001$ \\
Complications, $n$ (\%) & $7(3.6)$ & $118(60.5)$ & 0.034 \\
Surgery, $n,(\%)$ & $106(54.1)$ & $88(44.9)$ & 0.199 \\
I\&D at bedside, $n,(\%)$ & $37(18.9)$ & $76(38.8)$ & $<0.001$ \\
I\&D at OR, $n$ (\%) & $68(34.7)$ & $6(3.1)$ & 0.402 \\
Wound debridement at OR, $n$ (\%) & $9(4.6)$ & $8(4.1)$ & 0.300 \\
Re-infection within 30 days, $n,(\%)$ & $22(11.2)$ & 0.006 \\
\hline
\end{tabular}

ABSSSI acute bacterial skin and skin structure infection, $B M I$ body mass index, $B S I$ bloodstream infection, $I C U$ intensive care unit, $I D$ infectious disease, $I \leftrightarrow D$ incision and drainage, $I Q R$ interquartile range, $I V$ intravenous, $n$ number, $O R$ operating room, $S D$ standard deviation, $W B C$ white blood cell count

${ }^{a}>30 \%<$ High school education indicates patient resided in a zip code where $>30 \%$ of the population of has not completed high school

${ }^{\mathrm{b}}$ By zip code

c $N=207$

d Temperature $<35.6 \mathrm{C}$ or $>38.0 \mathrm{C}$

e WBC $>11 \times 10^{9}$ cells $/ 1$

${ }^{\mathrm{f}}$ Skin/skin structure specimen

g Clinical failure at discharge defined as persistent symptoms, need for a change in antibiotics (not including antibiotic de-escalation) or further surgical intervention

ABSSSI. African American race (aOR 2.18, 95\% CI 1.38, 3.4), elevated WBC (aOR 2.24, 95\% CI $1.41,3.55)$, and prior hospitalization (aOR, 2.07, 95\% CI 1.19, 3.0) were independently associated with severe infection. Although renal failure and polymicrobial ABSSSI had an increased association with severe infection $(\mathrm{aOR}$ of $2.26,95 \%$ CI $0.932,5.5$ and aOR of $1.87,95 \%$ CI $0.930,3.74$, respectively), these were not statistically significant.

\section{DISCUSSION}

In this study, we examined a population in urban Detroit, MI, at risk for ABSSSI and ABSSSI + BSI. Although risk factors have been evaluated in patients hospitalized for ABSSSI, there are limited studies on the risk for BSI in patients with CO-ABSSSI [9]. In addition, there are no investigations on the risk for BSI in patients with ABSSSI from an urban environment such as Detroit. It is important to evaluate inner-city populations because these populations often are of lower socioeconomic status and therefore are at risk because of limited access to appropriate medical care [26, 27]. These individuals may be more likely to delay seeking medical attention, which increases the risk of complications including hospitalization, surgery, relapse, and readmission [8, 28]. A delay in seeking medical attention for ABSSSI can also lead to the acquisition of BSI with severe complications including mortality $[11,28]$.

Several researchers have examined the relationship between patient characteristics and BSI in individuals with ABSSSI, which included the acquisition of MRSA from the community, older age, male gender, coronary heart disease, the presence of infected prosthetic devices, health care-associated infections, and abnormal WBC and vital signs $[6,9]$. However, these investigations included patients with hospital-acquired infection, making it difficult to extrapolate these findings to CO-ABSSSI + BSI. In addition, the contribution of socioeconomic factors was not examined. Consistent with previous investigations, we have also found that older age, male gender, abnormal temperature, and 

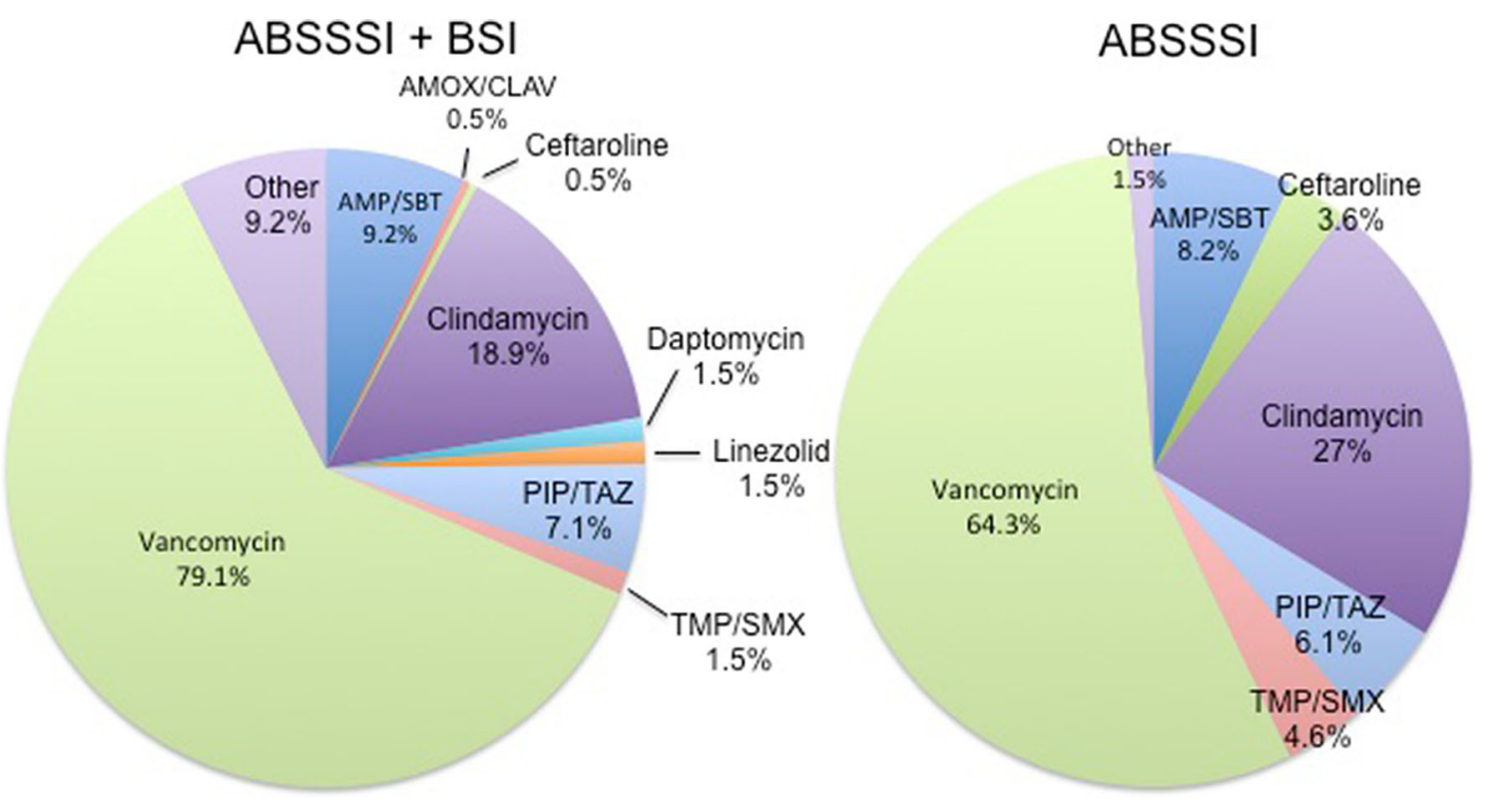

Fig. 1 Comparison of antibiotic treatment for cases vs. controls, Detroit, MI, 2010-2015. ABSSSI acute bacterial skin and skin structure infection, BSI blood stream infection, $A M O X / C L A V$ amoxicillin-clavulanate, $A M P /$

elevated WBC on hospital admission were significantly associated with ABSSSI + BSI. Among our study population, the presence of MRSA, prior hospitalization, prior MSSA infection, and $>30 \%$ with less than a high school education by zip code were additional risk factors. Although income and other sociodemographic characteristics had been previously evaluated for MRSA infections, the relationship between education level and ABSSSI + BSI had not been reported previously.

In the present study, a number of comorbid conditions were also found to be associated with ABSSSI + BSI including acute and chronic renal failure, hepatic disease, diabetes, hypertension, congestive heart failure, and intravenous drug abuse. Several of these factors had been previously reported. However, these studies focused only on hospitalized patients; therefore, the data may not be generalizable to patients who derived BSI in the community [9].

Abscess or abscess plus cellulitis appeared to have a protective effect against BSI. The most probable explanation for this finding may be related to routine medical intervention that
$S B T$ ampicillin-salbactam, PIP/TAZ piperacillin-tazobactam, TMP/SMX sulfamethoxazole-trimethoprimsulfamethoxazole

commonly includes incision and drainage of the abscess, which removes the focus of the infection and therefore probably protects the patients from BSI as a complication of ABSSSI [1, 10, 29].

As expected, a significantly higher percentage of patients with ABSSSI + BSI required an infectious disease consult, were admitted to the ICU, deemed a failure at time of discharge, and had a higher rate of mortality and reinfection within 30 days of discharge underscoring the need for early detection and optimized management in patients with ABSSSI + BSI.

As pertains to our second research question examining risk factors for severe ABSSSI defined as need for surgical intervention in the operating room, amputation, ICU admission, or severe sepsis, we found that African American race, elevated WBC on admission, prior hospitalization, and presence of abscess plus cellulitis were independently associated with severe infection. Other researchers have found that African Americans have significantly higher rates of skin and soft tissue infections compared with Caucasians [8]. In addition, African Americans 
Table 2 Logistic regression analysis of patient risk factors for ABSSSI + BSI

\begin{tabular}{lllll}
\hline Factor & Unadjusted OR & 95\% confidence interval & Adjusted OR & 95\% confidence interval \\
\hline Gender (male) & 1.74 & $1.16,2.61$ & 1.85 & $1.11,3.66$ \\
$>30 \%<$ High school education $^{\mathrm{a}}$ & 2.10 & $0.92,4.80$ & 2.91 & $0.98,8.64$ \\
Acute renal failure $^{\text {9 }}$ & 3.08 & $2.0,4.74$ & 2.08 & $1.18,3.66$ \\
IV Drug abuse $^{\text {Charlson Comorbidity Index }}$ & 1.98 & $1.16,3.39$ & 4.38 & $2.22,8.62$ \\
Hospitalization within 180 days & 3.24 & $1.18,1.42$ & 1.13 & $0.99,1.28$ \\
Abnormal temperature & 3.25 & $1.93,5.43$ & 2.41 & $1.24,4.70$ \\
Elevated WBC & 2.07 & $1.81,4.16$ & 2.86 & $1.66,4.93$ \\
Abscess & 2.75 & $0.432,1.01$ & 4.26 & $2.43,7.47$ \\
Abscess + cellulitis & 0.659 & $0.28,0.64$ & 0.220 & $0.11,0.46$ \\
\hline
\end{tabular}

Hosmer-Lemeshow $p=0.305$

ABSSSI: acute bacterial skin and skin structure infection; BSI: bloodstream infection; IV: intravenous; OR: odds ratio; WBC: white blood cell count

a $>30 \%<$ High school education indicates patient resided in a zip code where $>30 \%$ of population of has not completed high school

b Temperature $<35.6{ }^{\circ} \mathrm{C}$ or $>38.0^{\circ} \mathrm{C}$

${ }^{c}$ WBC $>11 \times 10^{9}$ cells $/ 1$

of low socioeconomic status have previously been found to have a higher rate of persistent infection, higher readmission rates, and increased infection complications [13, 28, 30]. However, the impact of race on severity of disease in patients with ABSSSI and ABSSSI + BSI had not been evaluated prior to the present study.

Patients in our study diagnosed with cellulitis without abscess had a significantly lower OR for severe disease while abscess plus cellulitis was associated with an increased risk of severe disease. Individuals with abscess are more likely to have surgical drainage as a part of their treatment intervention, which removes the foci of infection and improves the likelihood of antibiotic success $[1,10,29]$. This procedure likely prevents patients' localized infection (abscess) from spreading into the surrounding tissue causing cellulitis and potentially BSI. Individuals with abscess plus cellulitis already have an extensive infection that has spread into the surrounding tissues, which may explain the observed association with severe infection.
However, the fact the foci could still potentially be removed may at the same time explain the protective effect against BSI.

\section{Study Limitations}

This study had a retrospective study design and therefore was subject to all of the potential biases associated with this type of investigation. For example, it was possible that there were missing data or incomplete data recorded in the EMR. While it appeared that incomplete or missing data were minimal during the data collection, it is possible that some data were not recorded or were recorded incorrectly. Another potential limitation was the assumption that in patients with the diagnosis of cellulitis and BSI, the $S$. aureus identified in the bloodstream originated from the patient's cellulitis. Cellulitis is a deep-seated infection of the subcutaneous tissues of the skin, and therefore it is difficult to culture the pathogen causing disease. While $S$. aureus is a pathogen associated with cellulitis, 
Table 3 Logistic regression analysis of patient risk factors by severity

\begin{tabular}{lllll}
\hline Factor & \multicolumn{1}{l}{ Unadjusted OR } & 95\% confidence interval & Adjusted OR & 95\% confidence interval \\
\hline African American & 2.17 & $1.43,3.28$ & 2.18 & $1.38,3.44$ \\
Elevated WBC & 2.32 & $1.52,4.5$ & 2.24 & $1.41,3.55$ \\
Acute renal failure & 1.50 & $0.73,3.06$ & 2.26 & $0.932,5.49$ \\
Cellulitis & 0.155 & $0.087,0.31$ & 0.202 & $0.92,0.44$ \\
Abscess + cellulitis & 3.0 & $1.39,3.17$ & 1.65 & $1.03,2.65$ \\
Polymicrobial & 1.84 & $0.97,3.49$ & 1.86 & $0.930,3.74$ \\
Hospitalization within 180 days & 1.58 & $0.98,2.56$ & 2.07 & $1.193,3.59$ \\
\hline
\end{tabular}

Hosmer-Lemeshow $p=0.843$

$O R$ odds ratio, $W B C$ white blood cell count

${ }^{\text {a }}$ WBC $>11 \times 10^{9}$ cells $/ 1$

b Skin/skin structure specimen

other bacterial pathogens such as streptococci are also a frequent cause of cellulitis $[2,10]$. Therefore, although unlikely, cases of BSI where cellulitis without abscess was identified as the infection type may have been misclassified. Additionally, we evaluated the impact of race and ethnicity on severity of disease. The composite definition for severity included amputation. However, no amputations were recorded in the population cohort. It was possible that the exclusion of diabetic foot infections from this study is the most likely reason for the lack of amputations because surgical intervention including amputation is common among this population. Further, one of the objectives of this investigation was to determine whether sociodemographic characteristics were associated with ABSSSI + BSI. However, this information was derived by zip code that was provided through Federal census data. Information on education and level of income was not available on an individual basis; therefore, the information was limited. We observed that patient outcomes were poorer among those with bacteremia. We did not further investigate the impact of treatment factors, such as drug choice or time to effective therapy, because it was beyond the scope of our research objective; however, future studies investigating the impact of management strategies on outcomes specifically in ABSSI +BSI would be valuable.
Unfortunately, the time from symptom onset to presentation was not consistently documented, and therefore we were unable to analyze this factor. Lastly, the study was conducted on a population of mostly African American individuals who resided in Detroit or its immediate surrounding areas. Therefore, it is possible that the results of this investigation cannot be extrapolated to other metropolitan populations.

\section{CONCLUSION}

We have identified a number of patient characteristics, including sociodemographic factors such as level of education, that may contribute to BSI in patients with ABSSSI. In addition, several factors including African American race were associated with a higher degree of infection severity. According to these findings, there is a need to include sociodemographic characteristics when evaluating infection-related patient outcomes. Partnerships among community organizations, public health authorities, and intercity hospitals are needed to educate, screen, and identify individuals at risk to prevent these infections in this patient population. Further research is warranted to more fully characterize these risks and prevent these complications in this vulnerable patient population. 


\section{ACKNOWLEDGEMENTS}

Funding. No funding or sponsorship was received for this study or publication of this article.

Authorship. All named authors meet the International Committee of Medical Journal Editors (ICMJE) criteria for authorship for this article, take responsibility for the integrity of the work as a whole, and have given their approval for this version to be published.

Prior Presentation. This study was presented in part at IDWeek 2017, 4-8 October, San Diego, CA; Abstract 259.

Disclosures. Michael J. Rybak, Evan J. Zasowski, Sarah C.J. Jorgensen, Abdalhamid M. Lagnf, Susan L. Davis, Aaron B. Mendelsohn, and Vasileios Margaritis have nothing to disclose with respect to this study. At the time of the study, Evan J. Zasowski's affiliation was the Anti-Infective Research Laboratory, College of Pharmacy and Health Sciences, Wayne State University, Detroit, MI, USA. His current affiliation is Touro University, Vallejo, CA, USA.

Compliance with Ethics Guidelines. This study was approved by the institutional review boards (IRBs) at Walden University (IRB\# 0303767) and Wayne State University (IRB\# 010616M1E), with the latter serving as coordinating IRB for the DMC review committee and HFHS IRB. A waiver of informed consent was granted. The study was performed in accordance with the 1964 Helskinki Declaration and its later amendments.

Open Access. This article is distributed under the terms of the Creative Commons Attribution-NonCommercial 4.0 International License (http://creativecommons.org/licenses/ by-nc/4.0/), which permits any noncommercial use, distribution, and reproduction in any medium, provided you give appropriate credit to the original author(s) and the source, provide a link to the Creative Commons license, and indicate if changes were made.

\section{REFERENCES}

1. Moran GJ, Abrahamian FM, Lovecchio F, Talan DA. Acute bacterial skin infections: developments since the 2005 Infectious Diseases Society of America (IDSA) guidelines. J Emerg Med. 2013;44:e397-412.

2. Dryden MS. Complicated skin and soft tissue infection. $\mathrm{J}$ Antimicrob Chemother. 2010;65(3):35-44.

3. Edelsberg J, Taneja C, Zervos M, et al. Trends in US hospital admissions for skin and soft tissue infections. Emerg Infect Dis. 2009;15:1516-8.

4. Amin AN, Cerceo EA, Deitelzweig SB, Pile JC, Rosenberg DJ, Sherman BM. Hospitalist perspective on the treatment of skin and soft tissue infections. Mayo Clin Proc. 2014;89:1436-51.

5. Itani KM, Merchant S, Lin SJ, Akhras K, Alandete JC, Hatoum HT. Outcomes and management costs in patients hospitalized for skin and skin-structure infections. Am J Infect Control. 2011;39:42-9.

6. Tattevin P, Schwartz BS, Graber CJ, et al. Concurrent epidemics of skin and soft tissue infection and bloodstream infection due to community-associated methicillin-resistant Staphylococcus aureus. Clin Infect Dis. 2012;55:781-8.

7. Suaya JA, Eisenberg DF, Fang C, Miller LG. Skin and soft tissue infections and associated complications among commercially insured patients aged 0 64 years with and without diabetes in the US. PloS One. 2013;8:60057.

8. Ray GT, Suaya JA, Baxter R. Incidence, microbiology, and patient characteristics of skin and softtissue infections in a US population a retrospective population-based study. BMC Infect Dis. 2013;13:252.

9. Lipsky BA, Kollef MH, Miller LG, Sun X, Johannes RS, Tabak YP. Predicting bacteremia among patients hospitalized for skin and skin-structure infections: derivation and validation of a risk score. Infect Control Hosp Epidemiol. 2010;31:828-37.

10. Stevens DL, Bisno AL, Chambers HF, et al. Practice guidelines for the diagnosis and management of skin and soft tissue infections: 2014 update by the Infectious Diseases Society of America. Clin Infect Dis. 2014;59:e10-52.

11. van Hal SJ, Jensen SO, Vaska VL, Espedido BA, Paterson DL, Gosbell IB. Predictors of mortality in Staphylococcus aureus Bacteremia. Clin Microbiol Rev. 2012;25:362-86. 
12. Klevens RM, Morrison MA, Nadle J, et al. Invasive methicillin-resistant Staphylococcus aureus infections in the United States. JAMA. 2007;298:1763-71.

13. Zajacova A, Dowd JB, Aiello AE. Socioeconomic and race/ethnic patterns in persistent infection burden among US adults. J Gerontol A Biol Sci Med Sci. 2009;64:272-9.

14. Bassetti M, Trecarichi EM, Mesini A, et al. Risk factors and mortality of healthcare-associated and community-acquired Staphylococcus aureus bacteraemia. Clin Microbiol Infect. 2012;18:862-9.

15. Shurland S, Zhan M, Bradham DD, Roghmann MC. Comparison of mortality risk associated with bacteremia due to methicillin-resistant and methicillin-susceptible Staphylococcus aureus. Eur J Clin Microbiol Infect Dis. 2007;28:273-9.

16. Food and Drug Administration. Guidance for industry acute bacterial skin and skin structure infections: Developing drugs for treatment, US Department of Health and Human Services Food and Drug Administration Center for Drug Evaluation and Research(CDER). 2013. Retrieved Sept 10, 2015, from http://www.fda.gov/downloads/Drugs/ .../Guidances/ucm071185.pdf.

17. Micek ST, Hoban AP, Pham V, et al. Bacteremia increases the risk of death among patients with softtissue infections. Surg Infect. 2010;11:169-76.

18. Horan TC, Andrus M, Dudeck MA. CDC/NHSN surveillance definition of health care-associated infection and criteria for specific types of infections in the acute care setting. Am J Infect Control. 2008;36:309-32.

19. Harris PA, Taylor R, Thielke R, Payne J, Gonzalez N, Conde JG. Research electronic data capture (REDCap)-a metadata-driven methodology and workflow process for providing translational research informatics support. J Biomed Inform. 2009;42:377-81.

20. Levy MM, Fink MP, Marshall JC, et al. 2001 SCCM/ ESICM/ACCP/ATS/SIS international sepsis definitions conference. Crit Care Med. 2003;31:1250-6.
21. Fleiss JL, Lebin B, Myunghee CP. Statistical methods for rates and proportions. Hoboken: Wiley; 2003.

22. Carratala J, Roson B, Fernandez-Sabe N, et al. Factors associated with complications and mortality in adult patients hospitalized for infectious cellulitis. Eur J Clin Microbiol Infect Dis. 2003;22:151-7.

23. Lark RL, Saint S, Chenoweth C, Zemencuk JK, Lipsky BA, Plorde JJ. Four-year prospective evaluation of community-acquired bacteremia: epidemiology, microbiology, and patient outcome. Diagn Microbiol Infect Dis. 2001;41:15-22.

24. OpenEpi. Open source epidemiology statistics for public health: sample size calculation for unmatched case control study. Retrieved on July 23rd from: http://www.openepi.com/Menu/OE_Menu. htm. 2015.

25. Paul P, Pennell ML, Lemeshow S. Standardizing the power of the Hosmer-Lemeshow goodness of fit test in large data sets. Stat Med. 2013;32:67-80.

26. Khabbaz RF, Moseley RR, Steiner RJ, Levitt AM, Bell BP. Challenges of infectious diseases in the USA. Lancet. 2014;384:53-63.

27. Lantz PM, Pritchard A. Socioeconomic indicators that matter for population health. Prev Chronic Dis. 2010;7:A74.

28. Acosta CD, Knight M, Lee HC, Kurinczuk JJ, Gould JB, Lyndon A. The continuum of maternal sepsis severity: incidence and risk factors in a populationbased cohort study. PLoS One. 2013;8:e67175.

29. Pollack CV Jr, Amin A, Ford WT Jr, et al. Acute bacterial skin and skin structure infections (ABSSSI): practice guidelines for management and care transitions in the emergency department and hospital. J Emerg Med. 2015;48:508-19.

30. Morris DS, Rohrbach J, Sundaram LM, et al. Early hospital readmission in the trauma population: are the risk factors different? Injury. 2014;45:56-60. 